\title{
New-onset diabetes after transplant: Incidence, risk factors and outcome
}

\author{
S C Alagbe, ${ }^{1}$ MBBS, MPH, FWACP, MPhil, Cert Nephrology (SA) Phys; A Voster, ${ }^{2}$ BSc Hons, MSc; \\ R Ramesar, ${ }^{2}$ BSc Hons, MSc, PhD, Exec MBA; C R Swanepoel, ${ }^{1}$ MB ChB, MRCP, FRCP (Edin) \\ ${ }^{1}$ Division of Nephrology and Hypertension, Faculty of Health Sciences, University of Cape Town, South Africa \\ ${ }^{2}$ Division of Human Genetics, Faculty of Health Sciences, University of Cape Town, South Africa
}

Corresponding author: S C Alagbe (salagbe2001@yahoo.com)

\begin{abstract}
Background. The outcome of renal transplantation depends on achieving effective immunosuppression while minimising the consequences of such treatment. The occurrence of new-onset diabetes in the post-transplant period has been associated with several risk factors including some immunosuppressive medication. Better understanding of the clinical and genetic risk factors associated with new-onset diabetes after transplant (NODAT) could enable risk stratification of patients in the pre-transplant period, with the goal of applying measures that will reduce the incidence.

Objectives. To ascertain the incidence of and clinical and genetic risk factors that predispose to NODAT, and to examine its effect on the outcome of renal transplantation.

Methods. We performed a retrospective cohort review of all renal transplants at Groote Schuur Hospital, Cape Town, South Africa, between 2004 and 2008. Patients who were lost to follow-up or had pre-transplant diabetes or primary non-function were excluded. A subset of the cohort who gave informed consent was enlisted for genetic tests.

Results. We identified 111 patients who met the inclusion criteria. The incidence of NODAT was $18.0 \%$ ( $n=20$ patients). Risk factors for NODAT included age at transplant $(p=0.03)$, body weight $(p=0.04)$, treatment for acute cellular rejection $(p=0.02)$ and polycystic kidney disease as the cause of renal failure $(p=0.005)$. None of the genes investigated (TCF7L2 rs11196205, $r s 12255372$ and $r s 7903146$ and HNF1 $\beta$ $r s 1800575, r s 121918671$ and $r s 121918672)$ was found to be significantly associated with the risk of NODAT. The genotype frequencies for the single-nucleotide polymorphisms studied were closer (although not identical) to those reported for Caucasians than to those reported for the Yoruba (black) population in West Africa. Overall patient survival was $78 \%$ at five years, while graft survival was $72 \%$. There was no significant difference in patient or graft survival between the group with NODAT and the group without.

Conclusions. NODAT was common in renal transplant recipients. Some risk factors predate transplant and could be used to risk-stratify patients to determine appropriate risk-reduction strategies. The genetic determinants for NODAT in this population may differ from those reported elsewhere. NODAT had no impact on patient or graft survival in this cohort.
\end{abstract}

S Afr Med J 2017;107(9):791-796. DOI:10.7196/SAMJ.2017.v107i9.12258

Renal transplantation has gradually become commonplace since the first successful attempt in the 1950s, and is the preferred mode of renal replacement therapy for many patients with end-stage renal disease. The widespread use of this treatment modality is largely due to improvement in short-term outcome from $\sim 50 \%$ in the first year for the earliest attempts to $>90 \%$ in the first year in many centres around the world today. ${ }^{[1,2]}$ Much of this improvement is due to the introduction of new and more effective immunosuppressive agents since the 1980s, when cyclosporine was first used, as well as improved surgical techniques. ${ }^{[2,3]}$ However, together with the achievement of better immunosuppression came issues and consequences associated with such therapy, including infection, drug toxicity and metabolic complications such as new-onset diabetes. These also profoundly affect outcome. It is therefore evident that to achieve further improvements in the outcome of renal transplantation, it is necessary not only to prevent graft rejection by achieving effective immunosuppression but also to identify and if possible prevent or effectively manage the consequences of such therapy.

The occurrence of clinical hyperglycaemia in the post-transplant period has been recognised from the early days of renal transplantation. It was initially described as steroid diabetes, because steroids were the mainstay of transplant immunosuppression and have long been recognised as a cause of both transient hyperglycaemia and established diabetes mellitus in the general population. With the arrival of cyclosporine and newer immunosuppressive medications, as well as the increasingly widespread practice of transplantation, it soon became evident that diabetes in the post-transplant period was multifactorial, and new terms such as 'post-transplant diabetes mellitus' and 'newonset diabetes after transplant' (NODAT) were developed.

\section{Objectives}

To investigate the incidence of and clinical and genetic risk factors that may predispose to the occurrence of NODAT in renal allograft recipients at Groote Schuur Hospital, Cape Town, South Africa (SA).

\section{Methods}

We performed a retrospective cohort folder review of all patients who received a renal transplant and follow-up at our centre from 1 January 2004 to 31 December 2008, and were at least 18 years old at the time of the study. Patients were excluded if they had primary non-function of the allograft or were diabetic before the transplant. Information retrieved from the folders was recorded on a data collection sheet for further analysis. Data obtained included demographic details, body weight at transplant, type of underlying renal disease, duration and type of dialysis received, date of and age at transplant, number of previous transplants, family history of 
diabetes, and immunosuppressive protocol used. Laboratory data obtained included fasting plasma glucose, serum creatinine and total cholesterol before the transplant as well as serially after the transplant at 3,6 and 12 months and then annually thereafter until the patient reached an endpoint (return to dialysis or death) or was lost to follow-up, or until the end of the study period. The onset of diabetes was determined from the laboratory data (fasting plasma glucose $(\mathrm{FPG})>7 \mathrm{mmol} / \mathrm{L}$ or random plasma glucose $>11.1 \mathrm{mmol} / \mathrm{L}$ ) and clinical features.

\section{Genetic study}

Patients were enlisted for genetic tests if they gave written informed consent. The study was approved by the Groote Schuur Hospital/ University of Cape Town Research Ethics Committee in accordance with the Helsinki Declaration (ref. no. REC/REF 149/2009).

\section{DNA isolation}

Isolation of DNA from whole-blood samples was done using the 'salting out' method as described by Mullis and Faloona, ${ }^{[4]}$ with minor modifications. Specifically, the red blood cells were first separated out from the white blood cells (WBCs) that contain the DNA. The WBC membranes were then lysed with a protein denaturant (Proteinase K; Thermo Scientific, USA) and detergent (sodium dodecyl sulphate) to release the DNA from the nucleus. The protein content of the cell was precipitated out, separated from the DNA and discarded. The DNA was then precipitated using 100\% ethanol, washed and reconstituted. The DNA pellet was reconstituted by adding $100 \mu \mathrm{L}$ of DNA rehydration solution. DNA stock solutions were stored at $-20^{\circ} \mathrm{C}$.

\section{DNA quality and integrity}

The quality of the DNA samples obtained was assessed using various techniques, including spectrophotometry (to measure the DNA concentration and purity of the samples) using the Nanodrop 1000 spectrophotometer (Thermo Scientific, USA) and agarose gel electrophoresis (to determine the integrity of the DNA samples) using a $1 \%(\mathrm{w} / \mathrm{v})$ agarose gel (Lonza, Switzerland) with a final concentration of $0.5 \mu \mathrm{g} / \mathrm{ml}$. Ethidium bromide (Sigma, USA) DNA stain was incorporated into the gel, and electrophoresis was perfomed at $140 \mathrm{~V}$ for $30-40$ minutes. The DNA was visualised under ultraviolet light using the UVIPRO transilluminator (UVItec, UK).

\section{Gene annotation, primer design and polymerase chain reaction (PCR)}

Bioinformatic tools were used to annotate the genes of interest (TCF7L2- NG_012631.1 and HNF1 $\beta$ - NG_013019.1) with DNA variation features and aid experimental primer/assay design. The DNA sequences for TCF7L2 and HNF1 $\beta$ were taken from the genome browsers, National Centre for Biotechnology Information (NCBI), version 36 (http://www.ncbi.nlm.nih.gov), and Ensemble, version 54 (http://www.ensemble.org/index.html).

PCR techniques were used to amplify patients' DNA and to identify their genotypes for the various single-nucleotide polymorphisms (SNPs) studied.

\section{Statistical analysis}

The data were analysed using the SPSS statistical package, version 18.1 (IBM, USA) and R statistics. ${ }^{[5]}$ Continuous data were expressed as means, while discrete data were expressed as frequencies and percentages. The $\chi^{2}$ test and logistic regression model were used to test for statistical significance, and $p<0.05$ was chosen as the level of statistical significance. For the Kaplan-Meier survival curves, the initiation date was the date of transplant, and graft and patient survival was censored for loss to follow-up, completion of the study or outcome, i.e. return to dialysis or death with a functioning graft.

\section{Results}

Several clinical characteristics of the study population were analysed to determine whether they were associated with an increased risk of NODAT in this cohort. A higher age and weight at transplant, treatment for acute cellular rejection and polycystic kidney disease were found to be significantly associated with the risk of NODAT. Laboratory data such as serum cholesterol, serum creatinine at discharge and urine protein/creatinine ratio, as well as systolic and diastolic blood pressure, were similar in those with and without NODAT. Mean FPG at discharge was significantly higher in the group with NODAT. Other clinical parameters investigated were not associated with the risk of NODAT in this retrospective cohort (Table 1).

The proportion of subjects who developed NODAT and the time from the date of the transplant to the diagnosis of NODAT were analysed using a Kaplan-Meier curve. There was an incidence of $18.0 \%(20 / 111)$. Most cases of NODAT were diagnosed within the first 6 months after the transplant (Fig. 1).

The incidence of NODAT was assessed separately in the two main treatment arms (cyclosporine- v. tacrolimus-based immunosuppression) to determine whether either of the two drugs posed a greater risk of NODAT. Although the group treated with tacrolimus had slightly more cases of NODAT $(5 / 25,20.0 \%)$ than the group treated with cyclosporine $(15 / 86,17.4 \%)$, this difference was not statistically significant (Fig. 2).

Table 1. Clinical characteristics of the study population and the risk of NODAT

\begin{tabular}{|c|c|c|c|}
\hline Features & No NODAT & NODAT & $p$-value \\
\hline Age at transplant $(\mathrm{yr})$, mean & 37 & 44 & $0.03^{*}$ \\
\hline Weight at discharge $(\mathrm{kg})$, mean & 67 & 74 & $0.04^{*}$ \\
\hline \multicolumn{4}{|l|}{ Sex, $n(\%)$} \\
\hline Female & $34(77.3)$ & $10(22.7)$ & 0.2 \\
\hline Male & $57(85.1)$ & $10(14.9)$ & 0.2 \\
\hline \multicolumn{4}{|l|}{ Race, $n(\%)$} \\
\hline Mixed & $55(78.6)$ & $15(21.4)$ & 0.06 \\
\hline Black & $24(88.9)$ & $3(11.1)$ & 0.06 \\
\hline White & $12(85.7)$ & $2(14.3)$ & 0.06 \\
\hline Family history of DM, $n$ (\%) & $62(93.9)$ & $4(6.1)$ & 0.3 \\
\hline SBP (mmHg), mean & 133 & 135 & 0.7 \\
\hline DBP (mmHg), mean & 81 & 79 & 0.5 \\
\hline $\begin{array}{l}\mathrm{FBG} \text { at discharge }(\mathrm{mmol} / \mathrm{L}) \text {, } \\
\text { mean }\end{array}$ & 5.8 & 8.1 & $0.02^{*}$ \\
\hline Cholesterol (mmol/L), mean & 5.3 & 5.1 & 0.6 \\
\hline UPCR, mean & 0.05 & 0.05 & 0.8 \\
\hline $\begin{array}{l}\text { Creatinine at discharge } \\
(\mu \mathrm{mol} / \mathrm{L}) \text {, mean }\end{array}$ & 190 & 223 & 0.6 \\
\hline Donor type (CD), $n(\%)$ & $61(79.2)$ & $16(20.8)$ & 0.8 \\
\hline Tacrolimus use, $n(\%)$ & $18(78.3)$ & $5(2.7)$ & 0.1 \\
\hline Treatment for ACR, $n(\%)$ & $5(45.5)$ & $6(54.5)$ & $0.02^{*}$ \\
\hline Polycystic kidneys, $n$ (\%) & $3(27.3)$ & $8(72.7)$ & $0.005^{*}$ \\
\hline Dialysis type (PD), $n(\%)$ & $29(93.5)$ & $2(6.5)$ & 0.7 \\
\hline Dialysis duration (d), mean & 832 & 689 & 0.5 \\
\hline
\end{tabular}




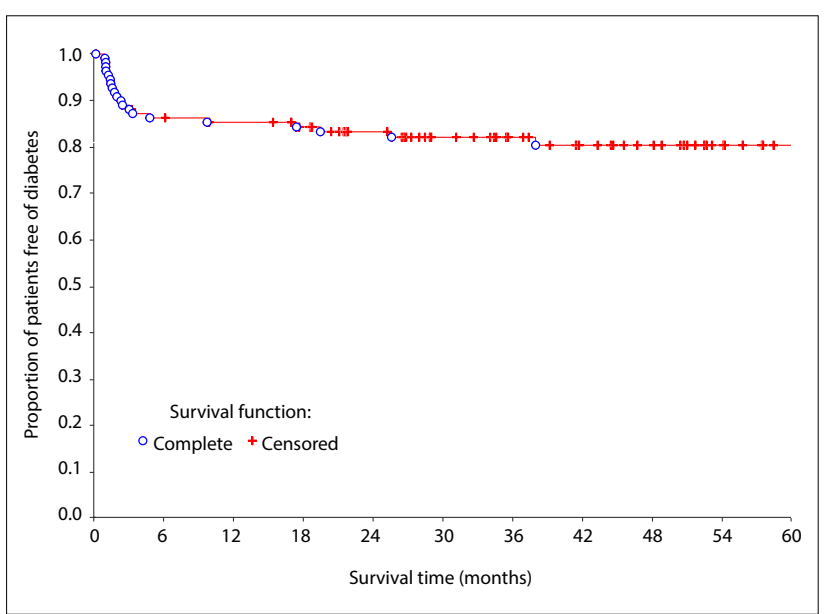

Fig. 1. Kaplan-Meier curve showing the incidence of NODAT over time. The blue circles represent patients who developed NODAT. (NODAT = newonset diabetes after transplant.)

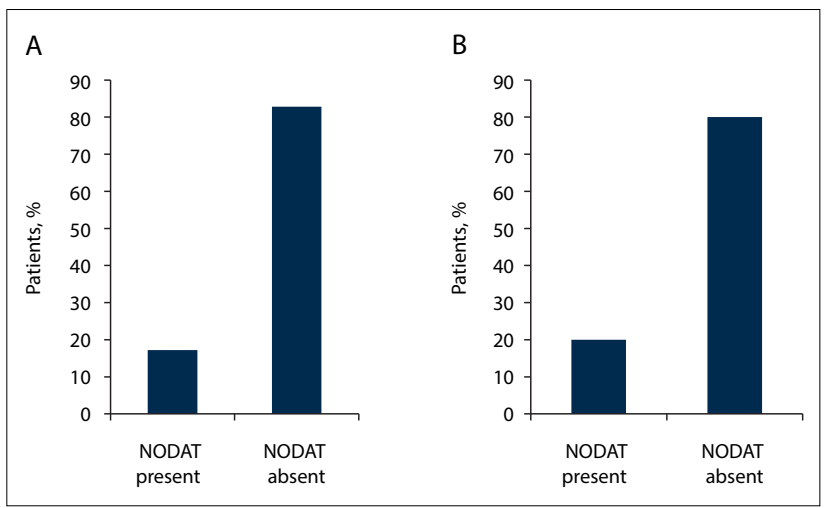

Fig. 2. Incidence of NODAT according to treatment arm $(A=$ cyclosporine; $B=$ tacrolimus). $($ NODAT $=$ new -onset diabetes after transplant.$)$

The time from date of transplant to diagnosis of NODAT was assessed separately in the two treatment arms (tacrolimus v. cyclosporine). Although the risk for NODAT was highest in the first 6 months for both treatment arms, all cases of NODAT in the tacrolimus treatment arm had occurred by 12 months, while the risk continued until 36 months for those treated with cyclosporine.

The impact of NODAT on graft survival was analysed using a Kaplan-Meier plot. Graft survival at 5 years was $72 \%$ (Fig. 3). There was no significant difference in graft survival between the patients with NODAT and those without $(p=0.4)$.

Patient survival over the study period was analysed using a Kaplan-Meier plot. The 5-year patient survival in this cohort was 78\% (Fig. 4). There was no significant difference in survival between the group with NODAT and the group without $(p=0.42)$.

In summary, the genotyping results (Table 2) showed no significant differences between the case and control groups when the frequencies of the SNPs selected for the study were compared. Logistic regression analysis was used to test for statistical significance. For comparison with published genotyping frequencies in other population groups, data were obtained from the NCBI database (http://www.ncbi.nlm.nih.gov). The genotype frequencies for the TCF7L2 SNPs $r s 12255372, r s 11196205$ and $r s 7903146$ in this SA population followed a distribution closer to that observed in the European Caucasian population than to that in the Yoruba (Nigerian) population. Genotyping frequencies for the SNPs in $H N F 1 \beta$ were not available on the public databases for comparison.

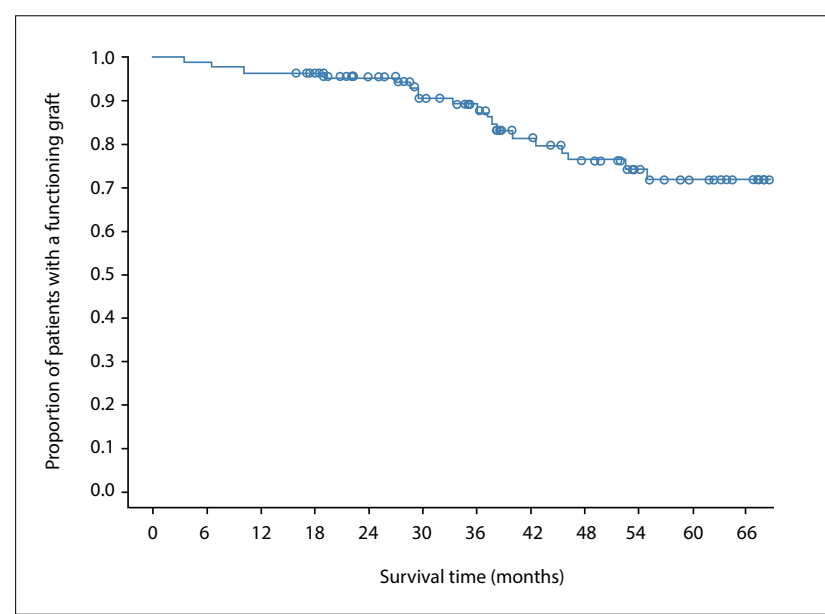

Fig. 3. Kaplan-Meier curve showing graft survival over time. The blue line represents survival function, while the blue circles represent an event (death, return to dialysis or re-transplant).

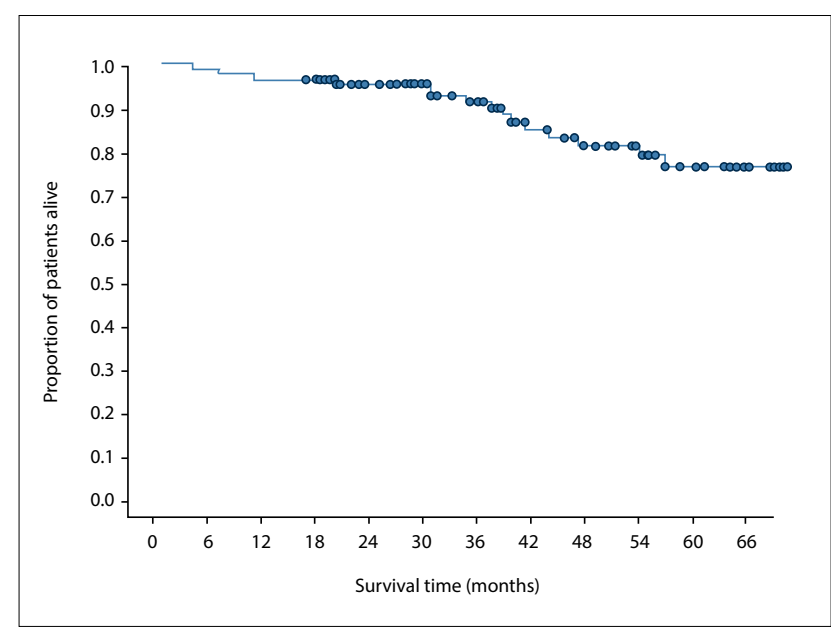

Fig. 4. Kaplan-Meier curve showing patient survival over time. The blue line represents survival function, while the blue circles represent an event (death).

\section{Discussion}

The incidence of NODAT in this study was $18.0 \%$ (Fig. 1). This is similar to the findings in another study, which used similar definitions for NODAT. ${ }^{[6,7]}$ Harrichund et al. ${ }^{[8]}$ reported a similar prevalence of $15.6 \%$ in Johannesburg. The age distribution of our patient cohort reflects a relatively young population, owing to the systematic exclusion of patients aged $>60$ years from the transplant programme in the public sector. Although the risk of NODAT continued throughout the duration of follow-up, most of the incident cases occurred within the first 6 months after the transplant (Fig. 1). This is similar to findings in several other studies, ${ }^{[7,9]}$ and supports the probability that transplant-related factors such as immunosuppressive medications, viral infections and the stress of surgery are major contributors to the risk of diabetes in the post-transplant period. The first few months after a transplant, when the risk of acute rejection is high, are characterised by high levels of exposure to immunosuppressive drugs. Extra doses of steroids increase the risk of viral and other opportunistic infections, making this a period of considerable vulnerability for renal transplant recipients.

The risk factors associated with NODAT in this study included age at transplant ( $>40$ years), higher weight at transplant, treatment for acute cellular rejection, and polycystic kidney disease (Table 1). 


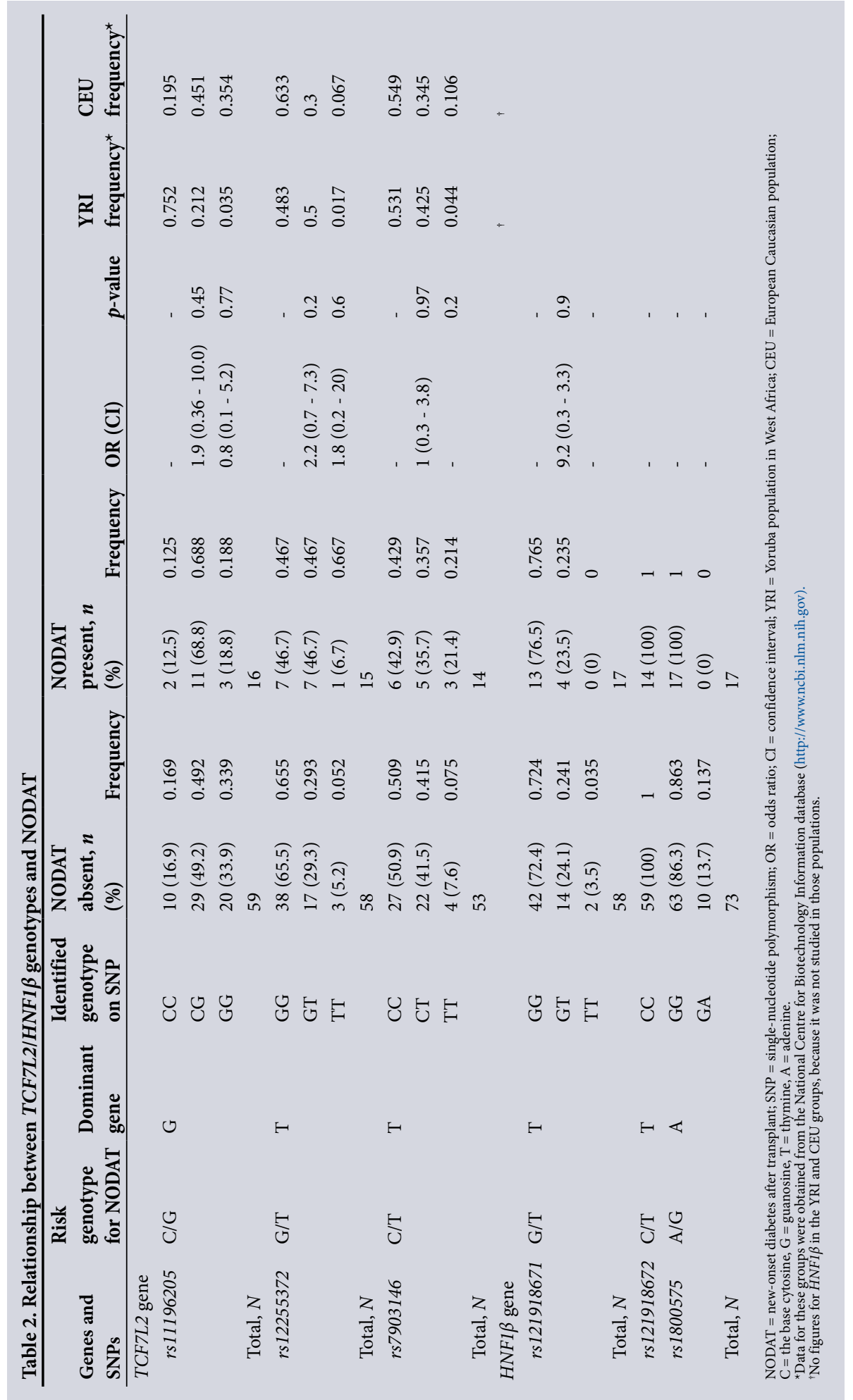

These findings are in keeping with those of other studies reporting that older age at transplant is significantly associated with an increased risk of NODAT. ${ }^{[7,10]}$ We know that the pathophysiology of post-transplant diabetes mimics that of type 2 diabetes mellitus in the general population. It is therefore not unexpected that they should body mass index (BMI)/body weight was associated with an increased risk of NODAT in several studies, ${ }^{[6,9,10]}$ while others found no significant association. This may reflect the different indices of body mass used in the studies, such as BMI v. weight, as well as the timing of measurement, e.g. before or immediately after the transplant or at the time of discharge, as the presence of fluid overload may have a considerable effect on weight at the different times. Furthermore, the number of overweight patients may be under-represented in some cohorts. We used weight at the time of discharge in our study, as this may be more representative of the dry weight in patients with a functioning graft than pre-transplant or immediate posttransplant weight.

Treatment for acute cellular rejection was found to be significantly associated with an increased risk of NODAT in this study (Table 1). Treatment for acute cellular rejection reflects an increase in the total dose of steroids used in these individuals, as all patients in the cohort received similar doses of oral steroids except for those with acute cellular rejection, who were all given extra pulses of high-dose methylprednisone. Several other studies have reported similar findings, showing that the risk of NODAT is directly related to the use of steroids ${ }^{[11,12]}$ This association is in agreement with established knowledge on the diabetogenic potential of steroids in the general population. However, we did not find a significant difference between cyclosporine and tacrolimus in terms of their contribution to the risk of NODAT (Table 1). Although there was a trend toward a higher rate of NODAT in the group treated with tacrolimus, it was not statistically significant (Fig. 2). This is similar to findings in some other studies, a few of which had similar methodology to ours. ${ }^{[6,13]}$ Several further studies, however, report a significantly greater role for tacrolimus in the causation of NODAT. ${ }^{[9,12]}$ Our finding may reflect the relatively small number of patients who received tacrolimus compared with cyclosporine in our cohort. It is also difficult to accurately tease out the contribution of each of the immusuppressive medications to the risk of NODAT; instead, the overall level of immunosuppression may be a simpler indicator of risk.

Polycystic kidneys as a cause of renal failure were also found to be significantly associated with an increased risk of NODAT in our cohort (Table 1), and several other studies have reported similar findings. ${ }^{[14-16]}$ Whether this association is due to an increased risk of maturity-onset diabetes of 
the young (MODY) type 5 (also known as 'renal cyst and diabetes' syndrome) in this group of patients is worth exploring. Mutations in the HNF1 $\beta$ gene, which is known to cause MODY type 5 , have also been shown to contribute to type 2 diabetes in some populations. ${ }^{[17-19]}$

A family history of diabetes was not significantly associated with an increased risk of NODAT, and nor were gender or race (Table 1). Although there was a trend to an increased risk in patients of mixed ancestry, this did not achieve statistical significance. The lack of a significant association between race and NODAT in this study may be due to the disproportionately high representation of people of mixed ancestry in this cohort compared with other racial groups.

Other clinical and biochemical characteristics such as type of donor (cadaver or live), blood pressure, serum cholesterol and urine protein/ creatinine ratios were similar in the NODAT and control groups.

We also investigated some possible genetic risk factors for NODAT. None of the three SNPs on the TCF7L2 gene investigated ( $r$ 11196205, $r s 12255372$ and $r s 7903146)$ was found to be significantly associated with the risk of NODAT (Table 2). A study among Hispanics did not find a significant association of polymorphisms in rs7903146 and rs12255372 with an increased risk of NODAT. ${ }^{[20]}$ Several studies, however, report a significant association between various SNPs on the TCF7L2 gene and the risk of NODAT. In Korea, a significant association between the risk of NODAT and polymorphisms in the TCF7L2 (rs7903146) gene has been reported. ${ }^{[21,22]}$ Another study reported a significant association between polymorphisms in the TCF7L2 rs7903146 gene only with late-onset post-transplant diabetes (later than 2 weeks) and in patients treated with tacrolimus. ${ }^{[23]}$ This may imply that the presence of a risk genotype in itself may not be sufficient to produce a clinical phenotype but requires the coexistence of other environmental determinants such as type and dose of immunosuppression. In our study, the fact that tacrolimus was used sparingly and only for specific indications may explain the lack of significant association between this gene and NODAT. Among the Zulu (black) people of KwaZulu-Natal, SA, a significant relationship was found between polymorphisms on the rs7903146 SNP on the TCF7L2 gene and diabetes in the general population. ${ }^{[24]}$ Similar work has yet to be performed among other racial groups in SA. The lack of a significant association between NODAT and the TCF7L2 gene in our study may also be explained by the fact that most of our cohort consisted of people of mixed ancestry. Furthermore, the number of SNPs studied was few, and it may be that the risk-carrying SNPs in this population are different from those we studied. The genotype frequencies in our cohort for the SNPs studied were closer (although not identical) to those reported for the European population and differed from those reported for the Yoruba population in West Africa. This again highlights the uniqueness of our cohort in terms of race, being neither purely black nor Caucasian.

Three SNPs in the HNF1 $\beta$ gene ( $r s 121918671, r s 121918672$ and rs1800575) were examined. Rs121918672 was homozygous in the entire cohort and could not be analysed further (Table 2). For $r s 1800575$, only one individual was heterozygous for the risk allele, and this also could not be investigated further (Table 2). For rs121918671, we found no significant relationship between carrying the risk allele and NODAT (Table 2). This may also be explained by population differences with regard to the disease-carrying SNPs on specific genes. This study has not been performed previously in our population. It may therefore imply that results from other populations with different characteristics from ours cannot be directly applied to our population.

We found no significant difference in graft (Fig. 3) or patient (Fig. 4) survival between the group with NODAT and the controls.
This differs from what has been reported in several other studies, and may be due to the relatively short duration of follow-up. Some studies have also reported no difference in outcome between subjects with NODAT and those without, especially in the short term. ${ }^{[25,26]}$ The mean serum creatinine level and glomerular filtration rate were also similar in the two groups (Table 1).

\section{Recommendations}

Insulin resistance has been identified as the mechanism by which weight gain increases the risk of type 2 diabetes in the general population. Could the mechanism in NODAT be the same? Do all the risk factors identified increase the incidence of NODAT via the same pathophysiological mechanism? Further studies need to be conducted to answer these questions. Despite a negative outcome on the study of genetic influence on NODAT, we nevertheless recommend an in-depth study of this factor with a larger sample size and a less skewed population.

\section{Conclusions}

NODAT is a common complication of renal transplantation. Some of the risk factors predate transplant and could be used to riskstratify patients and apply risk-reduction strategies such as lifestyle modification and use of less diabetogenic immunosuppressive protocols. Genotype frequencies for the SNPs studied differ from those reported for other populations, underscoring the genetic heterogeneity of our study population.

Acknowledgements. We gratefully acknowledge the contributions of Koliwe Dubula for help with DNA isolation, and Shareefa Dalvie and Motasim Badri for help with the statistical analysis.

Author contributions. SCA contributed to study conception and design, genetic study, data acquisition, analysis and interpretation, manuscript drafting and revision; AV contributed to design and co-ordination of the genetic study as well as manuscript drafting; RR contributed to design of the genetic study, data interpretation and manuscript drafting; and CRS contributed to study conception and design, data interpretation and revision of the manuscript. All authors read and approved the final manuscript.

Funding. None.

Conflicts of interest. None.

1. Collins AJ, Kasiske B, Herzog C, et al. Excerpts from the United States Renal Data System 2003 Annual Data Report: Atlas of end stage renal disease in the United States. Am J Kidney Dis 2003:41 (4 suppl 5):A57. https://doi.org/10.1016/S0272-6386(03)80001-X

2. Hariharan S, Johnson CP, Bresnahan BA, Taranto SE, McIntosh MJ, Stablein D. Improved graft survival after renal transplantation in the United States, 1988 to 1996. N Engl J Med 2009;342(9):605-612. https:/ doi.org/10.1056/NEJM200003023420901

3. Chakkera HA, Cherow GM, O'Hare AM, Amend WJ Jr, Gonwa TA. Regional variation in kidney transplant outcomes: Trends over time. Clin J Am Soc Nephrol 2009;4(1):152-159. https://doi. org/10.1056/NEJM200003023420901

4. Mullis KB, Faloona FA. Specific synthesis of DNA in vitro via a polymerase catalyzed chain reaction Methods Enzymol 1987:155:335-350. https://doi.org/10.1016/0076-6879(87)55023-6

R Core Team. R: A Language and Environment for Statistical Computing. Vienna, Austria: R Core Team, 2012. http://www.R-Project.org (accessed 10 August 2017).

6. Allamani M, Sennesael J, Vendemeulenbroucke E. Post transplantation diabetes mellitus: A long term retrospective cohort study. Transplant Proc 2010;42(10):4378-4383. https://doi.org/10.1016/j. transproceed.2010.07.009

7. Hadj AI, Adberrahim E, Ben Abdelghani KB, et al. Incidence and risk factors for post renal transplant diabetes mellitus. Transplant Proc 2011;43(2):568-571. https://doi.org/10.1016/j transproceed.2011.01.032

8. Harrichund P, Naicker S, Raal F, Becker P. New onset diabetes mellitus after renal transplantation. J Endocrinol Metab Diabetes S Afr 2008;13(3):98-104.

9. Baltar J, Ortega T, Ortega F, et al. Post transplantation diabetes mellitus: Prevalence and risk factors. Transplant Proc 2005:37(9):3817-3818. https://doi.org/10.1016/j.transproceed.2005.09.197

10. Boudreaux $J$. The impact of cyclosporine and combination immunosuppression on the incidence of post transplant diabetes in renal allograft recipients. Transplantation 1987;44(3):375-381. https://doi. post transplant diabetes in renal allograft
org/10.1097/00007890-198709000-00010

11. Hjelmesaeth J, Hartmann A, Kofstad J, et al. Glucose intolerance after renal transplantation depends Helmesaeth J, Hartmann A, Kofstad J, et al. Glucose intolerance after renal transplantation depends
upon prednisolone dose and recipient age. Transplantation 1997;64(7):979-983. https://doi. org/10.1097/00007890-199710150-00008 
12. Luan FL, Steffick DE, Ojo AO. New onset diabetes mellitus in kidney transplant recipients discharged on steroid free immunosuppression. Transplantation 2011;91(3):334-341. https://doi.org/10.1097/ TP.0b013e318203c25f

13. Guerra G, Ciancio G, Gaynor JJ, et al. Randomized trial of immunosuppressive regimens in renal transplantation. J Am Soc Nephrol 2011;22(9):1758-1768. https://doi.org/10.1681/ASN.2011010006

14. De Mattos AM, Olyaei AJ, Prather JC, Golconda MS, Barry JM, Norman DJ. Autosomal dorminant polycystic kidney disease as a risk factor for diabetes mellitus following renal transplantation. Kidney Int 2005;67(2):714-720. https://doi.org/10.1111/j.1523-1755.2005.67132.x

15. Jacquet A, Pallet N, Kessler M, et al. Outcomes of renal transplantation in patients with autosomal dominant polycystic kidney disease: A nation wide longitudinal study. Transpl Int 2011;24(6):582-587. https://doi.org/10.1111/j.1432-2277.2011.01237.x

16. Razeghi E, Heydarian P, Amerian M, Pourmand G. The risk factors for diabetes mellitus after kidney transplantation. Saudi J Kidney Dis Transpl 2010;21(6):1038-1043.

17. Furuta $\mathrm{H}$, Furuta $M$, Sanke $T$, et al. Nonsense and missense mutations in the human hepatocyte nuclear factor 1 beta gene (TCF2) and their relation to type 2 diabetes in Japanese. J Clin Endocrinol Metab 2002;87(8):3859-3863. https://doi.org/10.1210/jcem.87.8.8776

18. Bonnycastle LL, Willer CJ, Conneely KN, et al. Common variants in maturity onset diabetes of the young genes contribute to risk of type 2 diabetes in Finns. Diabetes 2006;55(9):2534-2540. https://doi. org/10.2337/db06-0178

19. Winckler W, Weedon MN, Graham RR, et al. Evaluation of common variants in the six known maturity onset diabetes of the young (MODY) genes for association with type 2 diabetes. Diabetes 2007;56(3):685693. https://doi.org/10.2337/db06-0202

20. Yang J, Hutchinson II, Shah T, Min DI. Genetic and clinical risk factors of new onset diabetes after transplantation in Hispanic kidney transplant recipients. Transplantation 2011;91(10):1114-1119. https:// doi.org/10.1097/TP.0b013e31821620f9
21. Kang ES, Kim MS, Kim YS, et al. A variant of the transcription factor 7 like 2 (TCF7L2) gene and the risk of post transplantation diabetes mellitus in renal allograft recipients. Diabetes Care 2008;31(1):63-68. https://doi.org/10.2337/dc07-1005

22. Kang ES, Kim MS, Kim CH, et al. Association of common type 2 diabetes risk gene variants and post transplantation diabetes mellitus in renal allograft recipients in Korea. Transplantation2009;88(5):693-698. transplantation diabetes mellitus in renal allogra
https://doi.org/10.1097/TP.0b013e3181b29c41

23. Kurzawski M, Dziewanowski K, Kedzierska K, Wajda A, Lapczuk J, Drozdzik M. Association of transcription factor 7 like 2 (TCF7L2) gene polymorphism with post transplant diabetes mellitus in kidney transplant patients medicated with tacrolimus. Pharmacol Rep 2011;63(3):826-833. https://doi. org/10.1016/S1734-1140(11)70595-3

24. Pirie F, Motala A, Pegoraro R, Paruk I, Govender T, Rom L. Variants in PPARG, KCNJ11, TCF7L2, FTO and HHEX genes in South African subjects of Zulu descent with type 2 diabetes. Afr J Diabetes Med 2010;july:12-16.

5. Kiberd M, Panek R, Kiberd BA. New onset diabetes mellitus post kidney transplantation. Clin Transplant 2006;20(5):634-639. https://doi.org/1010.2215/CJN.05360709

26. Kuo HT, Sampaio MS, Vincenti F, Bunnapradist S. Associations of pretransplant diabetes mellitus, new onset diabetes after transplant, and acute rejection with transplant outcomes: An analysis of the Organ Procurement and Transplant Network/United Network for Organ Sharing (OPTN/UNOS) database. Am J Kidney Dis 2010;56(6):1127-1139. https://doi.org/10.1053/i.aikd.2010.06.027 